\title{
Can Sex be A Predictor of Survival and Treatment Benefit in T1-3NOM0 colorectal Cancer Patients? A Population-Based Analysis
}

Bin Ma ( $D$ mabin0326cmu@163.com )

Liaoning Cancer Institute and Hospital https://orcid.org/0000-0002-0727-4536

Yongmin Li

Liaoning Cancer Institute and Hospital

Qingkai Meng

Liaoning Cancer Institute and Hospital

\section{Research}

Keywords: Colorectal cancer, Sex, Overall survival, Radiotherapy, SEER dataset

Posted Date: August 19th, 2020

DOI: https://doi.org/10.21203/rs.3.rs-61000/v1

License: (c) (i) This work is licensed under a Creative Commons Attribution 4.0 International License.

Read Full License 


\section{Abstract}

Background: Our aim was to assess predictive effect of sex on survival and treatment benefit of T13NOMO CRC.

Methods: This study population was assembled from the SEER dataset. Between 2004 and 2016, patients diagnosed with malignant primary CRC who underwent primary tumour resection were included.

Results: First, we found that females had better OS than males and that sex could be an independent factor of survival in T1-3NOMO colon cancer (regardless of right/left-sided tumour) and T1-2N0M0 rectal cancer (each $\mathrm{HR}<1$, each $\mathrm{P}<0.05$ ). In addition, though a significant $\mathrm{OS}$ benefit was not observed in T3NOMO rectal cancer in the overall analysis $(P=0.183)$, females benefited more than males among T3N0M0 rectal cancer patients who underwent postoperative chemotherapy $(P<0.001)$. However, there was no significant OS difference between male and femaleT3NOMO rectal cancer patients among those who did not receive postoperative chemotherapy $(P=0.634)$. In subgroup analyses of T3N0M0 patients, a similar tendency of survival and the effect of postoperative chemotherapy were observed regardless of whether preoperative radiotherapy was performed. Furthermore, we found that females had a survival benefit over males at all ages, among patients of the white race and among T1-T3 adenocarcinoma CRC patients (each $H R<1$, each $\mathrm{P}<0.001$ ).

Conclusion: Sex was proven to be an independent prognostic factor in T1-3N0M0 colon cancer and T12 NOMO rectal cancer. Females are more likely to benefit from postoperative chemotherapy than males with T3NOMO rectal cancer. Our research suggests that sex could be considered a factor in practical clinical treatment and prognosis evaluation for T1-3N0M0 rectal cancer.

\section{Introduction}

Cancer is the main global public health problem and is also the second primary cause of death in the United States of America [1]. Colorectal cancer (CRC) is one of the most common malignant tumours in the United States. In the male population, CRC is the third most common cancer after prostate cancer and lung cancer, with an estimated 78,300 new cases (9\% of all new cancers) in 2020. In the female population, $\mathrm{CRC}$ is the third most common cancer after breast cancer and lung cancer. It is estimated that there will be 69,650 new cases ( $8 \%$ of all new cancers) in 2020 . CRC also has a high death rate. CRC is the third most common cause of cancer death in males, with a total of 28,630 deaths ( $9 \%$ of all cancers) expected in 2020. Likewise, CRC is the third most common cause of cancer death among females, with 24,570 deaths ( $9 \%$ of all cancers) expected in 2020 . Although the morbidity and mortality of CRC rank third in both males and females in the estimated data, there are some differences between the sexes. In the vast majority of cancers, there are some differences between males and females in morbidity and prognosis, which is the reason why cancer data of males and females are separated.

The influence of sex on CRC has also been a hot topic in recent years. Several studies have shown that sex is an independent prognostic factor for CRC and that females generally have longer survival times 
than males [2-8]. Compared to males of all ages, females are less likely to have CRC [9-10]. In fact, their risk is equivalent to that of males 4 to 8 years younger [11]. There are several probable explanations that sex differences in CRC extend to environmental influence, tumour biology, and therapeutic response [12]. Hormones may be one reason. Postmenopausal hormone use has been linked to a $40 \%$ reduction in CRC in the Women's Health Initiative trial [13]. The link between hormone replacement therapy and colon cancer prevention is the same as in former reports [14-15]. The biological features of tumours may be different between males and females. There is also evidence that women are more likely to develop rightsided colon cancer with microsatellite instability [16-17]. Furthermore, some reports suggest that females present at a more advanced stage of cancer [18] on average. All aforementioned differences should be taken into account as confounding factors.

Therefore, females are more often hormone carriers and more often have right-sided colon cancer and more advanced stages of cancer. We selected patients aged 60 years and over (menopause) and patients with stage T1-4NOM0 (early local tumours) for analysis after stratification of the primary tumour site. Our aim was to assess the prognostic effect of sex on survival after removal of known confounding factors.

\section{Materials And Methods}

\section{Patients}

Between 2004 and 2016, patients diagnosed with malignant primary CRC were included in the study. The disease was defined by International Classification of Diseases for Oncology, third edition (ICD-0-3) codes (18.0, 18.2-18.7, 19.9 and 20.9). All patients who were diagnosed with CRC at or above the age of 60 underwent primary tumour resection. The patients were excluded from this study population if they (1) died within 30 days after tumour resection; (2) had Tis, N1, N2 or M1 cancer; (3) had other cancers before; or (4) lacked records of pathological stage, follow-up or histological subtype. These cases were organized by SEER's coding schemes. On the basis of the primary location of the tumour, patients were divided into three groups. Right-sided colon cancers were defined as those arising from the caecum (C18.0), ascending colon (C18.2), hepatic flexure of the colon (C18.3) or transverse colon (C18.4); left-sided colon cancer was defined as those arising from the splenic flexure of colon (C18.5), descending colon (C18.6), and sigmoid colon (C18.7); rectum cancers were defined as those developing from the rectosigmoid junction (C19.9) or rectum (C20.9).

\section{Data Collection}

This study population was assembled from the National Cancer Institute's Surveillance, Epidemiology, and End Results (SEER) Program. SEER data include cancer cases from different locations and sources across the United States. Only a limited number of registration agencies have been collected since 1973. The dataset continues to broaden by including more geographic areas and demographic groups. It cover approximately $28 \%$ of the U.S. population and includes data from various geographic areas, including incidence, prevalence, mortality, population-based variable quantities, main characteristics of the tumour, and other attributes. SEER*Stat software version 8.3.6 was used to gather the main data. 


\section{Statistical Analysis}

The chi-square test was used to compare the baseline characteristics of males and females. Overall survival outcomes were estimated with the Kaplan-Meier (KM) method, and the differences between survival were compared by the log-rank test. Multivariable Cox regression was used to calculate hazard ratios (HRs) and 95\% confidence intervals (Cls). All analyses were double-sided, and a $\mathrm{P}$ value lower than 0.05 was deemed statistically significant. All analyses were performed using the statistical software STATA 8.0 (STATA Inc, College Station, TX).

\section{Results}

\section{Demographic characteristics of male and female CRC}

We obtained a dataset that included 70,836 cases of CRC in males and 69,705 cases of CRC in females. The characteristics of CRC and the chi-square test for comparison of male and female CRC are shown in Table 1. We found some significant differences in age, race, histological grade, chemotherapy, marital status, histology, radiation, $\mathrm{pT}$ category and primary site (each $\mathrm{P}<0.001$ ). Compared with males, females more often hadage $\geq 75$ years ( $55.1 \%$ vs. $44 \%$ ), right-sided colon cancer ( $56.8 \%$ vs. $45.4 \%)$, and pT $2-4$ cancer $(75.5 \%$ vs. $72 \%)$. 
Table 1

Characteristics of males and females CRC

\begin{tabular}{|c|c|c|c|}
\hline & Males & Females & \\
\hline & $n(\%)$ & $n(\%)$ & $\mathrm{P}$ \\
\hline Age, years & & & $<0.001$ \\
\hline $60-64$ & 12235(17.3) & $8918(12.8)$ & \\
\hline $65-69$ & 13988(19.7) & 10927(15.7) & \\
\hline $70-74$ & 13443(19.0) & 11481(16.5) & \\
\hline $75-79$ & $12780(18.0)$ & 12792(18.4) & \\
\hline $80-84$ & 10394(14.7) & 12578(18.0) & \\
\hline$>84$ & 7996(11.3) & 13009(18.7) & \\
\hline Race & & & $<0.001$ \\
\hline Black & $6443(9.1)$ & $6878(9.9)$ & \\
\hline White & $58791(83.0)$ & $57573(82.6)$ & \\
\hline Other & $5271(7.4)$ & $5008(7.2)$ & \\
\hline Unknown & $331(0.5)$ & $246(0.4)$ & \\
\hline Histological grade & & & $<0.001$ \\
\hline Well & $8575(12.1)$ & 7815(11.2) & \\
\hline Moderately & $50272(71.0)$ & 47877(68.7) & \\
\hline Poorly & $6625(9.4)$ & $8961(12.9)$ & \\
\hline Undifferentiated & 894(1.3) & 1348(1.9) & \\
\hline Unknown & $4470(6.3)$ & $3704(5.3)$ & \\
\hline Chemotherapy & & & $<0.001$ \\
\hline Performed & $9607(13.6)$ & 7157(10.3) & \\
\hline None/Unknown & $61229(86.4)$ & $62548(89.7)$ & \\
\hline Marital status & & & $<0.001$ \\
\hline Single & 7275(10.3) & $6826(9.8)$ & \\
\hline Married & $46879(66.2)$ & $26637(38.2)$ & \\
\hline
\end{tabular}

Abbreviations: AC, adenocarcinoma; MC, Mucinous adenocarcinoma; SRCC, Signet ring cell carcinoma. 


\begin{tabular}{|c|c|c|c|}
\hline & Males & Females & \\
\hline Widowed & 13158(18.6) & $32404(46.5)$ & \\
\hline Unknown & $3524(5.0)$ & $3838(5.5)$ & \\
\hline Histology & & & $<0.001$ \\
\hline AC & $64160(90.6)$ & 61381(88.1) & \\
\hline MC & $5018(7.1)$ & 6195(8.9) & \\
\hline SRCC & $254(0.4)$ & $352(0.5)$ & \\
\hline Other & $1404(2.0)$ & $1777(2.5)$ & \\
\hline Radiotherapy & & & $<0.001$ \\
\hline None/Unknown & $64560(91.1)$ & 65739(94.3) & \\
\hline Before surgery & 2133(3.0) & $1568(2.2)$ & \\
\hline After surgery & $4143(5.8)$ & $2398(3.4)$ & \\
\hline pT category & & & $<0.001$ \\
\hline T1 & 19866(28.0) & $17060(24.5)$ & \\
\hline $\mathrm{T} 2$ & 14954(21.1) & 15142(21.7) & \\
\hline T3 & $31654(44.7)$ & $32009(45.9)$ & \\
\hline $\mathrm{T} 4$ & $4362(6.2)$ & $5494(7.9)$ & \\
\hline Primary site & & & $<0.001$ \\
\hline Right-sided colon & $32175(45.4)$ & $39569(56.8)$ & \\
\hline Left-sided colon & 20701(29.2) & $17051(24.5)$ & \\
\hline Rectum & $17960(25.4)$ & 13085(18.8) & \\
\hline
\end{tabular}

\section{Influence of sex on OS in $\mathrm{T}_{\text {any }} \mathrm{N}_{0} \mathrm{M}_{0}$}

There was a significant difference in survival between males with CRC and females with CRC in the T1 category $(P<0.001$, Fig. 1-A). There were similar significant differences in the $T 2$ and $T 3$ categories $(P<$ 0.001, Fig. 1-B; $P<0.001$, Fig. 1-C). In the T4 category, we discovered no difference in survival between male CRC and female CRC ( $P=0.784$, Fig. 1-D). In consideration of the above outcomes, we held T4 CRC out of our analyses. The multivariable Cox regression (Table 2) showed that sex was an independent prognostic factor $(\mathrm{HR}=0.716 ; 95 \% \mathrm{Cl}=0.703-0.730 ; \mathrm{P}<0.001)$. 
Table 2

Prognostic factors in COX proportional hazard model

Univariate Analysis

$H R$ $95 \% \mathrm{Cl}$

Gender

Male

Female

Age, years

$60-64$

65-69

$70-74$

$75-79$

$80-84$

$>84$

Race

Black

White

Other

Unknown

Histological grade

Well

Moderately

Poorly

Undifferentiated

Unknown

Chemotherapy

Performed

None/Unknown
1

0.926

$0.910-0.942$
$0.712 \quad 0.680-0.746$

$0.134 \quad 0.092-0.194$

1

$1.127 \quad 1.096-1.158$

$1.317 \quad 1.270-1.365$

$1.292 \quad 1.192-1.399$

$1.020 \quad 0.975-1.066$
Multivariate Analysis

HR $\quad 95 \% \mathrm{Cl} \quad \mathrm{P}$

$<0.001$

$<0.001$

1

$0.716 \quad 0.703-0.730$
$<0.001$

1

$1.371 \quad 1.314-1.429$

$1.913 \quad 1.838-1.990$

$2.786 \quad 2.681-2.894$

$3.980 \quad 3.831-4.134$
$6.105 \quad 5.874-6.346$
6.106
5.883-6.337

$<0.001$

$<0.001$

1

$\begin{array}{llll}0.987 & 0.958-1.017 & 0.832 & 0.808-0.857\end{array}$

$0.658 \quad 0.628-0.689$

0.160

$0.111-0.232$

$<0.001$

$<0.001$

1

1.026

0.997-1.056

$1.102 \quad 1.062-1.144$

$1.115 \quad 1.029-1.209$

1.127

1.077-1.178

$<0.001$

$<0.001$

1

$1.116 \quad 1.070-1.165$

Abbreviations: AC, adenocarcinoma; MC, Mucinous adenocarcinoma; SRCC, Signet ring cell carcinoma; HR, Hazard ratio; $\mathrm{Cl}$, Confidence interval. 


\begin{tabular}{|c|c|c|c|c|c|c|}
\hline \multirow[b]{2}{*}{ Marital status } & \multicolumn{3}{|c|}{ Univariate Analysis } & \multicolumn{3}{|c|}{ Multivariate Analysis } \\
\hline & & & $<0.001$ & & & $<0.001$ \\
\hline Single & 1 & & & 1 & & \\
\hline Married & 0.806 & $0.782-0.832$ & & 0.752 & $0.728-0.776$ & \\
\hline Widowed & 1.259 & $1.220-1.299$ & & 0.961 & $0.930-0.992$ & \\
\hline Unknown & 0.893 & $0.850-0.938$ & & 0.823 & $0.783-0.865$ & \\
\hline Histology & & & $<0.001$ & & & $<0.001$ \\
\hline$A C$ & 1 & & & 1 & & \\
\hline MC & 1.198 & $1.162-1.234$ & & 1.073 & $1.040-1.106$ & \\
\hline SRCC & 1.238 & $1.089-1.406$ & & 1.054 & $0.926-1.199$ & \\
\hline Other & 0.823 & $0.767-0.884$ & & 0.968 & $0.901-1.040$ & \\
\hline Radiotherapy & & & $<0.001$ & & & $<0.001$ \\
\hline None/Unknown & 1 & & & 1 & & \\
\hline Before surgery & 0.958 & $0.907-1.011$ & & 1.118 & $1.049-1.191$ & \\
\hline After surgery & 0.779 & $0.745-0.814$ & & 0.958 & $0.901-1.019$ & \\
\hline pT category & & & $<0.001$ & & & $<0.001$ \\
\hline T1 & 1 & & & 1 & & \\
\hline $\mathrm{T} 2$ & 1.187 & $1.157-1.217$ & & 1.059 & $1.031-1.087$ & \\
\hline T3 & 1.448 & $1.418-1.479$ & & 1.272 & $1.243-1.302$ & \\
\hline Primary site & & & $<0.001$ & & & $<0.001$ \\
\hline Right colon & 1 & & & 1 & & \\
\hline Left colon & 0.918 & $0.900-0.938$ & & 1.105 & $1.082-1.129$ & \\
\hline Rectum & 0.894 & $0.875-0.914$ & & 1.176 & $1.146-1.207$ & \\
\hline
\end{tabular}

\section{Influence of sex on OS after stratification by T category and primary site}

T1 category 
In right-sided colon cancer, there was no significant difference in survival between male CRC and female CRC $(\mathrm{P}<0.066$, Fig. 2-A), but we discovered significant differences on multivariable Cox regression (Supplemental Table 1), and sex was shown to be an independent prognostic factor ( $\mathrm{HR}=0.739,95 \% \mathrm{Cl}$ $=0.696-0.785, P<0.001$, Fig. 3 ). In left-sided colon and rectal cancer, there were significant differences in survival between male $C R C$ and female $C R C(P<0.001$, Fig. 2-B; $P<0.001$, Fig. $2-C)$. We also saw the result upon multivariable Cox regression (Supplemental Table 1). Sex was also proven to be an independent prognostic factor in this analysis $(\mathrm{HR}=0.730,95 \% \mathrm{Cl}=0.705-0.756, \mathrm{P}<0.001 ; \mathrm{HR}=0.688,95 \% \mathrm{Cl}=$ $0.639-0.740, \mathrm{P}<0.001$, Fig. 3).

\section{T2 category}

Within right-sided colon, left-sided colon or rectum cancers, we saw significant differences in survival between male and female patients (respectively, $P<0.001$, Fig. 2-D; $P<0.001$, Fig. 2-E; $P=0.005$, Fig. 2-F). We also discovered the same significant difference in multivariable Cox regression (Supplemental Table 2), where sex was again proven to be an independent prognostic factor $(\mathrm{HR}=0.713,95 \% \mathrm{Cl}=$ $0.675-0.753, \mathrm{P}<0.001 ; \mathrm{HR}=0.680,95 \% \mathrm{Cl}=0.624-0.740, \mathrm{P}<0.001 ; \mathrm{HR}=0.713,95 \% \mathrm{Cl}=0.658-0.773, \mathrm{P}$ $<0.001$, Fig. 3).

\section{T3 category}

We saw significant differences in survival between male patients and female patients with right-sided colon or left-sided colon cancer (respectively, $P<0.001$, Fig. 2-G; $P<0.001$, Fig. $2-H$ ). We discovered the same significant differences in multivariable Cox regression (Supplemental Table 3), which again showed that sex was an independent prognostic factor $(\mathrm{HR}=0.721,95 \% \mathrm{Cl}=0.696-0.746, \mathrm{P}<0.001 ; \mathrm{HR}=0.708$, $95 \% \mathrm{Cl}=0.674-0.745, \mathrm{P}<0.001)$. In the rectum, there was no significant difference in survival between male $C R C$ and female $C R C(P=0.183$, Fig. $2-1)$. We did not perform multivariable Cox regression on these data. It is apparent that sex is not an independent prognostic factor in rectal cancer.

\section{Influence of sex and postoperative chemotherapy on OS in rectum of T3}

Taking all tumours of the T3 category (Fig. 4-A), patients with postoperative chemotherapy had a better survival benefit than individuals without postoperative chemotherapy among females $(P<0.001)$ or males $(P<0.001)$. We also found that females had a better survival benefit than males in all tumours of the T3 category with postoperative chemotherapy $(P<0.001)$. There were no significant differences in survival between males and females without postoperative chemotherapy $(P=0.634)$.

For tumours with a postoperative pathological stage of T3NOM0, the preoperative clinical stage is often more advanced and may generally be locally advanced. These patients are also often treated with preoperative radiotherapy. Therefore, we performed a subgroup analysis for patients with and without preoperative radiotherapy. 
Among patients with T3 tumours who underwent preoperative radiotherapy (Fig. 4-B), those who also underwent postoperative chemotherapy had a better survival benefit than individuals without postoperative chemotherapy in both the female $(P=0.001)$ and the male sex $(P<0.001)$. We found an apparent difference, though without statistical significance, in survival between males and females with postoperative chemotherapy $(P=0.069)$. There were no significant differences in survival between males and females without postoperative chemotherapy $(P=0.226)$.

Among patients with T3 tumours who underwent preoperative radiotherapy (Fig. 4-C), those who underwent postoperative chemotherapy had a better survival benefit than individuals without postoperative chemotherapy in both the female $(P=0.001)$ and the male sex $(P<0.001)$. We also found that females had a better survival benefit than males with postoperative chemotherapy $(P<0.001)$. There were no significant differences in survival between males and females without postoperative chemotherapy $(P=0.618)$.

\section{Influence of sex on OS stratified by age/race/histology}

In six different age groups (supplemental Table 4), we found that females had a better survival benefit than males regardless of age and $T$ category (each $H R<1$, each $P<0.001$ ). In patients of the white race (supplemental Table 4), we found that females had a better survival benefit than males in the T1, T2 and T3 categories $(\mathrm{HR}=0.933,95 \% \mathrm{Cl}=0.897-0.970, \mathrm{P}<0.001 ; \mathrm{HR}=0.910,95 \% \mathrm{Cl}=0.875-0.947, \mathrm{P}<0.001$; $\mathrm{HR}=0.935,95 \% \mathrm{Cl}=0.911-0.959, \mathrm{P}<0.001)$. In several groups of different histological types (supplemental Table 4), we found that females had a survival benefit over males among AC patients in the T1, T2 and T3categories (respectively, $\mathrm{HR}=0.926,95 \% \mathrm{Cl}=0.893-0.960, \mathrm{P}<0.001 ; \mathrm{HR}=0.895,95 \%$ $\mathrm{Cl}=0.861-0.930, \mathrm{P}<0.001 ; \mathrm{HR}=0.902,95 \% \mathrm{Cl}=0.880-0.926, \mathrm{P}<0.001)$.

\section{Discussion}

Cancer is a major global public health problem and is also the second primary cause of death in the United States [1]. There are many differences between males and females in morbidity, mortality, prognosis, and biological behaviour for most cancers. The sex differences in cancer survival have attracted great attention because they may be a signal of basic biological differences between males and females in cancer pathogenesis and treatment response, which can be incorporated into management strategies. Therefore, sex differences are an essential research direction in tumour research. Interestingly, females seem to have better outcomes in most tumours, such as low morbidity, low mortality, and better prognosis. Moreover, a systematic review indicated that female patients also often have a survival advantage over male patients in many cancers [19]. Among the sex differences in some cancer manifestations, researchers have found that some may be related to male occupational and environmental exposures [20]. Nevertheless, in many circumstances, despite the control of environmental and genetic factors, the sex differences found in many cancers remain unexplained [21-22]. Our study focussed on the prognostic impact of sex on early local CRC. CRC is one of the most common malignant tumours in the U.S. It is the third most common tumour in both male and female patients. The estimated new cases in 2020 will be 78,300 in males and 69,650 in females. Among all tumours, the mortality rate 
of CRC ranks third among males and females. The data for 2020 projected deaths are 28,630 male patients and 24,570 female patients. Although the morbidity and mortality of CRC rank third in both male and female patients, there are some differences in their morbidity, mortality and prognosis.

The influence of sex on CRC has also been a hot topic in recent years. In 2001, a German study surveyed nearly 900 patients and concluded that being female was related to improved long-term survival in rectal cancer [6]. In 2003, a similar study evaluated 3,200 cases and found that females had a survival benefit in CRC [5]. More studies have indicated that sex is an independent prognostic factor in CRC, and females usually live longer than males [2-8]. Other studies indicated that females improved OS compared with males [3, 5, 23-24]. Compared to males of all ages, females are less likely to have CRC [9-10]. In fact, their risk is equivalent to that of males 4 to 8 years younger [11]. Thus, the effect of sex on CRC is well recognized. Although a large number of studies have shown that the female prognosis is better than male prognosis in many cases, there were many confounding factors that resulted in the better outcomes of women than men in these studies.

Therefore, the purpose of our research is to see whether the female superiority over males remains after controlling for confounding factors. There are several explanations for the sex differences in CRC, including environmental influence, tumour biology, and therapeutic response. First, hormones may be one reason. Postmenopausal hormone use has been linked to a 40\% reduction in CRC in the Women's Health Initiative trial [13]. This link between hormone replacement therapy and colon cancer prevention is in line with former reports [14-15]. Therefore, we believe that oestrogen may be a confounding factor, so our study cohort included only menopausal women. Menopause is usually defined as 12 months of amenorrhea in females over 45 years of age. It usually starts between 45 and 55 years of age, with an average age of 50.5 years [25]. However, there are women who go into menopause after age 55 [26]. Based on the above, we only selected patients aged 60 years and older to form our study cohort to ensure that all female patients were postmenopausal. Next, the biological features of tumours may be different between males and females. There is evidence that females are more likely to develop microsatelliteunstable phenotypes of right-sided tumours [7,27]. Furthermore, some reports suggest that females present at a more advanced stage of cancer [18]. Consequently, we selected early local CRC of stage T14 NOMO for analysis after stratification of the primary tumour site. Some studies have shown that sex, age, and ethnicity play an important role in the OS of CRC patients. A review of the literature showed that we analysed the largest cohort of colorectal cancer patients, and sex and age had an important impact on the OS of CRC patients. Therefore, we also conducted a detailed analysis stratified by both race and age.

In that analysis, we still found that females had better survival than males with T1-3NOMO colon cancer and T1-2N0M0 rectal cancer after taking away the effects all known confounding factors. However, there was no difference in survival between sexes among T3NOMO rectum cancer patients. For rectum tumours with a postoperative pathological stage of T3NOMO, the preoperative clinical stage is often more advanced and may generally be a locally advanced stage. These patients are often treated with preoperative neoadjuvant treatment. For rectum cancer of the PT3NOMO stage, the recommendation of 
the NCCN guidelines [28] is adjuvant therapy or observation. Adjuvant therapy is often recommended for T3rectal cancer with prognostic risk factors. Therefore, we also performed a subgroup analysis of patients with and without preoperative radiotherapy. As a result, we found the benefits of female sex among T3NOMO rectum cancer patients who underwent postoperative chemotherapy, regardless of whether preoperative radiotherapy was performed. Moreover, the results indicate that the T3NOMO rectum cancer patients who underwent postoperative chemotherapy had a survival benefit over individuals without postoperative chemotherapy, regardless of sex. Females more often have tumours with microsatellite instability. This reason, as suggested in many studies, could be that females benefit more from chemotherapy $[17,29]$. However, all of these studies were confined to more advanced CRC, and there are few studies about the influence of sex on chemotherapy in early-stage tumours (T3NOMO). Although it is generally accepted that females have a better survival than males, we did not find a survival benefit of females with T3colorectal cancer. A significant survival benefit was obtained in rectum cancer patients with postoperative chemotherapy, which also confirmed that women can benefit more from postoperative chemotherapy in early rectal cancer (T3NOMO). Therefore, in addition to the existence of risk factors, female sex can also be considered an indicator for postoperative chemotherapy in T3NOMO rectum cancer.

Although this research is based on a large number of samples, our research should be interpreted on the background of some limitations, some of which are intrinsic in the design of retrospective studies. Our results may not be representative of young patients with colorectal cancer. To exclude the effects of oestrogen on the results, we included only colorectal cancer patients aged 60 years and older. Information on radiotherapy and chemotherapy was 'None/Unknown', so we cannot rule out that some of these people had undergone radiotherapy or chemotherapy. Many significant clinical factors, predictive and prognostic biomarkers (e.g., microsatellite instability status), and behavioural differences (e.g., smoking, alcohol, diet, exercise and physical activity) were not available. In addition, there was no information in the SEER database about menopausal status or use of hormone therapy. Thus, there may have been some postmenopausal patients who were still taking oestrogen. Despite these limitations, our large-scale population-based research may make our conclusions more persuasive.

In conclusion, we found that females have a survival benefit over males among patients with T1-3NOM0 colon cancer and T1-2NOMO rectal cancer. Sex was also proven to be an independent prognostic factor in T1-3NOMO colon cancer and T1-2NOMO rectal cancer. Females are more likely to benefit from postoperative adjuvant chemotherapy than males with T3NOMO rectal cancer. Our research suggests that sex could be considered a factor in practical clinical treatment and prognosis evaluation for stage T1$3 \mathrm{NOMO}$ rectal cancer. Especially in clinical decision-making on postoperative adjuvant therapy for T3NOMO rectal cancer, female sex can also be considered an indicator for postoperative adjuvant therapy.

\section{Conclusion}

\section{Perspectives and significance}


In summary, based on the SEER database, we investigated predictive effect of sex on survival and treatment benefit of T1-3NOMO CRC. First, we found that females had better OS than males and that sex could be an independent factor of survival in T1-3NOMO colon cancer (regardless of right/left-sided tumour) and T1-2NOMO rectal cancer. In addition, though a significant OS benefit was not observed in T3N0M0 rectal cancer in the overall analysis $(P=0.183)$, females benefited more than males among T3N0M0 rectal cancer patients who underwent postoperative chemotherapy $(P<0.001)$. In brief囚Females are more likely to benefit from postoperative chemotherapy than males. In the clinical environment, sex could be considered a factor in practical clinical treatment and prognosis evaluation for T1-3NOMO rectal cancer.

\section{Abbreviations}

CRC: colorectal cancer; SEER: Surveillance, Epidemiology, and End Results; OS: Overall survival; HR: Hazard ratio; Cl: Confidence interval; pT category: Postoperative pathological T category; AC: Adenocarcinoma; MC: Mucinous adenocarcinoma; SRCC: Signet ring cell carcinoma.

\section{Declarations}

\section{Ethics approval and consent to participate}

Not applicable

\section{Consent for publication}

Not applicable

\section{Availability of data and materials}

The dataset generated and analyzed during the current study is available in the National Cancer Institute's Surveillance, Epidemiology, and End Results SEER repository [https://seer.cancer.gov/]. This study obtained the permission to access files.

\section{Competing interests}

The authors have declared that no conflicts of interest exist.

\section{Funding}

This work was supported by National Science Foundation of China (Grant/Award Number: 81902383), the Doctoral Scientific Research Startup Foundation of Liaoning Province (Grant/Award Number: 2019BS-146) and Natural Science Foundation of Liaoning Province (No.20180550485). Revitalizing Liaoning Talents Program (Grant/Award Number: XLYC1907004).

\section{Authors' contributions}


Bin Ma contributed to the conception of the study; Yongmin Li contributed significantly to analysis and manuscript preparation; Bin Ma performed the data analyses and wrote the manuscript; Qingkai Meng helped perform the analysis with constructive discussions.

\section{Acknowledgements}

Not applicable

\section{References}

1. Siegel RL1. Miller KD1, Jemal A1. Cancer statistics, 2020. CA Cancer J Clin. 2020 Jan;70(1):7-30. doi: 10.3322/caac.21590. Epub 2020 Jan 8.

2. Paulson EC, Wirtalla C, Armstrong K, et al. Gender influences treatment and survival in colorectal cancer surgery. Dis Colon Rectum. 2009;52(12):1982-91.

3. Koo JH, Jalaludin B, Wong SK, et al. Improved survival in young women with colorectal cancer. Am J Gastroenterol. 2008;103(6):1488-95.

4. van Leeuwen BL, Pahlman L, Gunnarsson U, et al. The effect of age and gender on outcome after treatment for colon carcinoma. A population-based study in the Uppsala and Stockholm region. Crit Rev Oncol Hematol. 2008;67(3):229-36.

5. McArdle CS, McMillan DC, Hole DJ. Male gender adversely affects survival following surgery for colorectal cancer. Br J Surg. 2003;90(6):711-5.

6. Wichmann MW, Muller $\mathrm{C}$, Hornung HM, et al. Gender differences in long-term survival of patients with colorectal cancer. Br J Surg. 2001;88(8):1092-8.

7. Cheung WY, Shi Q, O'Connell M, et al. The predictive and prognostic value of sex in early-stage colon cancer: a pooled analysis of 33,345 patients from the ACCENT database. Clin Colorectal Cancer. 2013;12(3):179-87.

8. Quirt JS, Nanji S, Wei X, et al. Is there a sex effect in colon cancer? Disease characteristics, management, and outcomes in routine clinical practice. Curr Oncol. 2017;24(1):e15-23.

9. Farquhar CM, Marjoribanks J, Lethaby A, Lamberts Q, Suckling JA. Long term hormone therapy for perimenopausal and postmenopausal women. Cochrane Database Syst Rev 2005:CD 004143.

10. Nelson RL, Dollear T, Freels S, Persky V. The relation of age, race, and gender to the subsite location of colorectal carcinoma. Cancer. 1997;80:193-7.

11. Brenner $\mathrm{H}$, Hoffmeister $\mathrm{M}$, Arndt $\mathrm{V}$, Haug $\mathrm{U}$. Gender differences in colorectal cancer: implications for age at initiation of screening. Br J Cancer. 2007;96:828-31.

12. Hendifar A, Yang D, Lenz F, Lurje G, Pohl A, Lenz C, Ning Y. Wu Zhang, and Heinz-Josef Lenz. Gender Disparities in Metastatic Colorectal Cancer Survival. Clin Cancer Res. 2009 Oct 15;15(20):6391-7.

13. Chlebowski RT, Wactawski-Wende J, Ritenbaugh C, et al. Estrogen plus progestin and colorectal cancer in postmenopausal women. N Engl J Med. 2004;350:991-1004. 
14. Newcomb PA, Storer BE. Postmenopausal hormone use and risk of large-bowel cancer. J Natl Cancer Inst. 1995;87:1067-71.

15. Mandelson MT, Miglioretti D, Newcomb PA, Harrison R, Potter JD. Hormone replacement therapy in relation to survival in women diagnosed with colon cancer. Cancer Causes Control. 2003;14:979-84.

16. Ashktorab H, Smoot DT, Carethers JM, et al. High incidence of microsatellite instability in colorectal cancer from African Americans. Clin Cancer Res. 2003;9:1112-7.

17. Elsaleh H, Joseph D, Grieu F, Zeps N, Spry N, lacopetta B. Association of tumour site and sex with survival benefit from adjuvant chemotherapy in colorectal cancer. Lancet. 2000;355:1745-50.

18. Woods SE, Basho S, Engel A. The influence of gender on colorectal cancer stage: the state of Ohio, 1996-2001. J Womens Health (Larchmt). 2006;15:877-81.

19. Molife R, Lorigan P, MacNeil S. Gender and survival in malignant tumours. Cancer Treat Rev. 2001;27:201-9.

20. Ferguson MK, Skosey C, Hoffman PC, Golomb HM. Sex-associated differences in presentation and survival in patients with lung cancer. J Clin Oncol. 1990;8:1402-7.

21. Edgren G, Liang L, Adami HO, Chang ET. Enigmatic sex disparities in cancer incidence. Eur J Epidemiol. 2012;27:187-96.

22. Dorak MT, Karpuzoglu E. Gender differences in cancer susceptibility: An inadequately addressed issue. Front Genet. 2012;3:268.

23. Chapuis $\mathrm{PH}$, Dent $\mathrm{OF}$, Fisher $\mathrm{R}$, et al. A multivariate analysis of clinical and pathological variables in prognosis after resection of large bowel cancer. Br J Surg. 1985;72(9):698-702.

24. Newland RC, Dent OF, Lyttle MN, et al. Pathologic determinants of survival associated with colorectal cancer with lymph node metastases. A multivariate analysis of 579 patients. Cancer. 1994;73(8):2076-82.

25. Nichols HB, Trentham-Dietz A, Hampton JM, et al. From menarche to menopause: trends among US Women born from 1912 to 1969. Am J Epidemiol. 2006;164:1003-11.

26. McKinlay S, Brambilla D, Posner J. The normal menopause transition. Maturitas. 1992;14:103-15.

27. Meguid R, Slidell M, Wolfgang $C$, Chang D, Ahuja N. Is there a difference in survival between rightversus left-sided colon cancers? Ann Surg Oncol. 2008;15:2388-94.

28. Rectal Cancer. Version 2.2019, NCCN Clinical Practice Guidelines in Oncology [www.nccn.org/patients].

29. Quirt JS, Nanji S, Wei X. Is there a sex effect in colon cancer? Disease characteristics, management, and outcomes in routine clinical practice. Curr Oncol. 2017 Feb;24(1):e15-23.

\section{Perspectives and significance.}

31. In summary, based on the SEER database, we investigated predictive effect of sex on survival and treatment benefit of T1-3NOMO CRC. First, we found that females had better OS than males and that sex could be an independent factor of survival in T1-3NOMO colon cancer (regardless of right/leftsided tumour) and T1-2N0M0 rectal cancer. In addition, though a significant OS benefit was not 
observed in T3NOMO rectal cancer in the overall analysis $(P=0.183)$, females benefited more than males among T3NOMO rectal cancer patients who underwent postoperative chemotherapy $(P<$ 0.001). In brief,Females are more likely to benefit from postoperative chemotherapy than males. In the clinical environment, sex could be considered a factor in practical clinical treatment and prognosis evaluation for T1-3NOM0 rectal cancer.

\section{Figures}
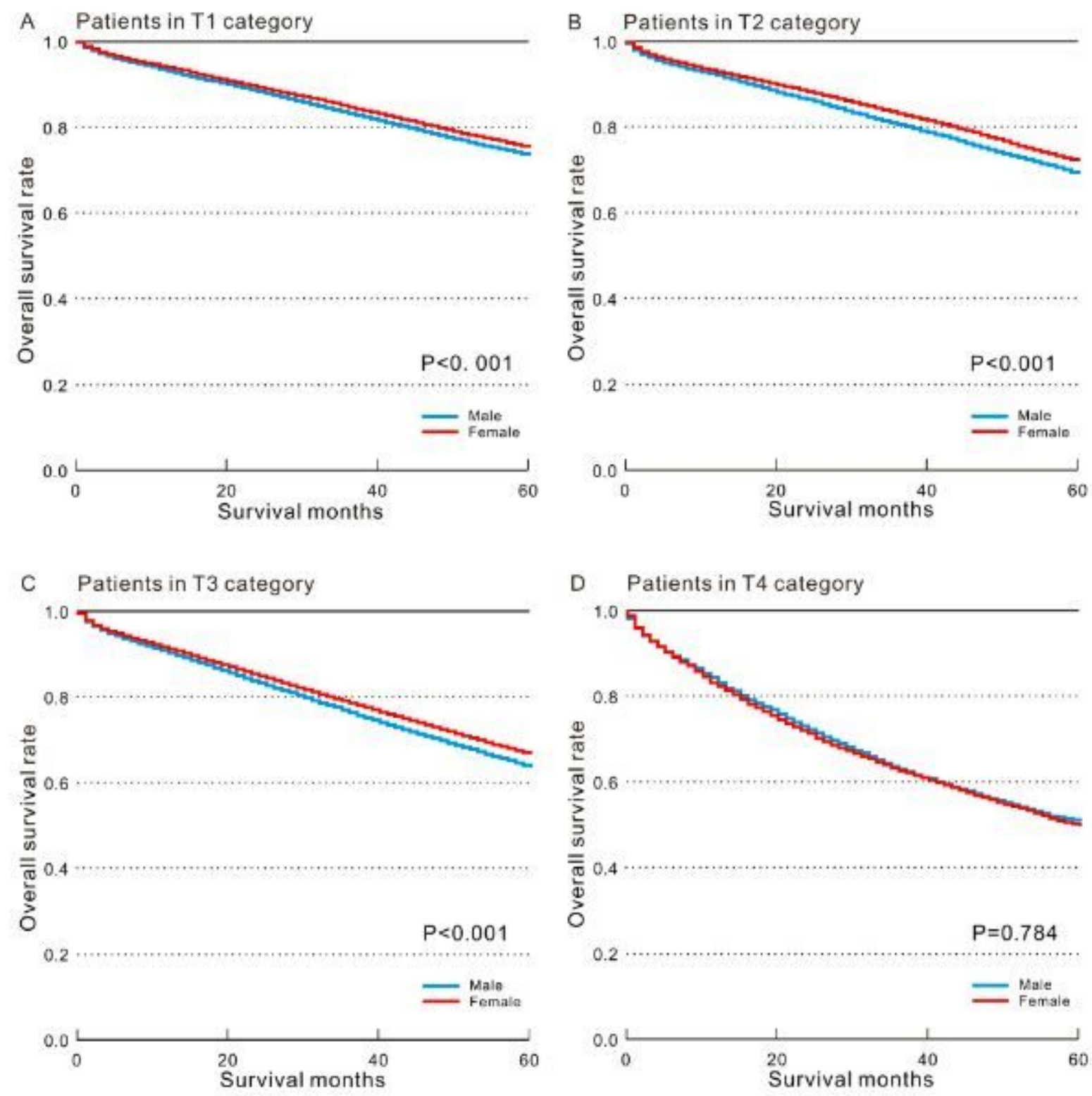

\section{Figure 1}

Kaplan-Meier comparison of overall survival among patients with males and females stratified by $T$ category. (A) Patients in T1 category; (B) Patients in T2 category ; (C) Patients in T3 category; (D) Patients in T1 category. 

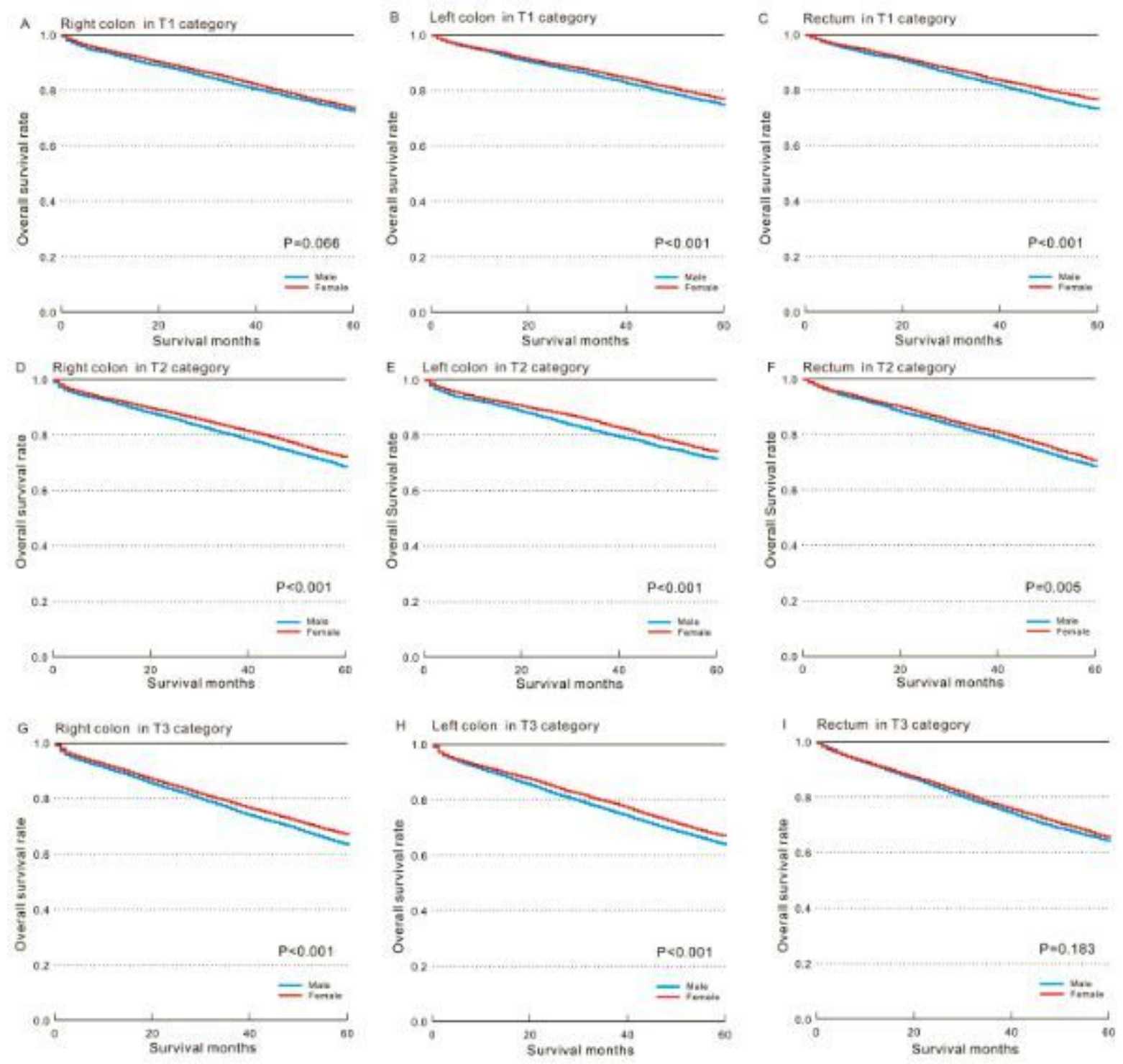

\section{Figure 2}

Kaplan-Meier comparison of overall survival among patients with males and females stratified by $T$ category and primary site. (A) Right colon in T1 category; (B) Left colon in T1 category; (C) Rectum in T1 category; (D) Right colon in T2 category; (E) Left colon in T2 category; (F) Rectum in T2 category; (G) Right colon in T3 category; $(\mathrm{H})$ Left colon in T3 category; (I) Rectum in T3 category. 
A Female vs Male for patients in T1 category

Hazard ratio $(95 \% \mathrm{Cl})$

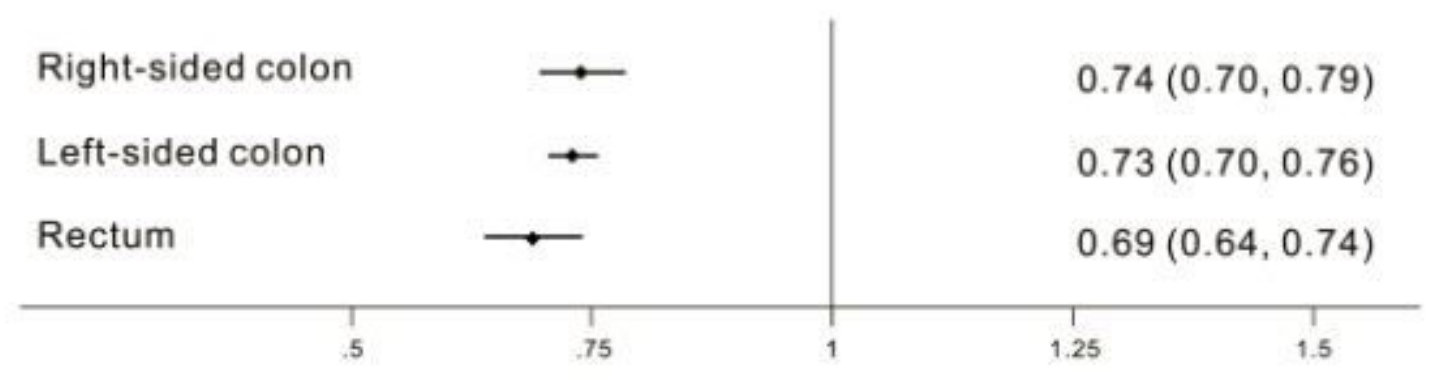

B

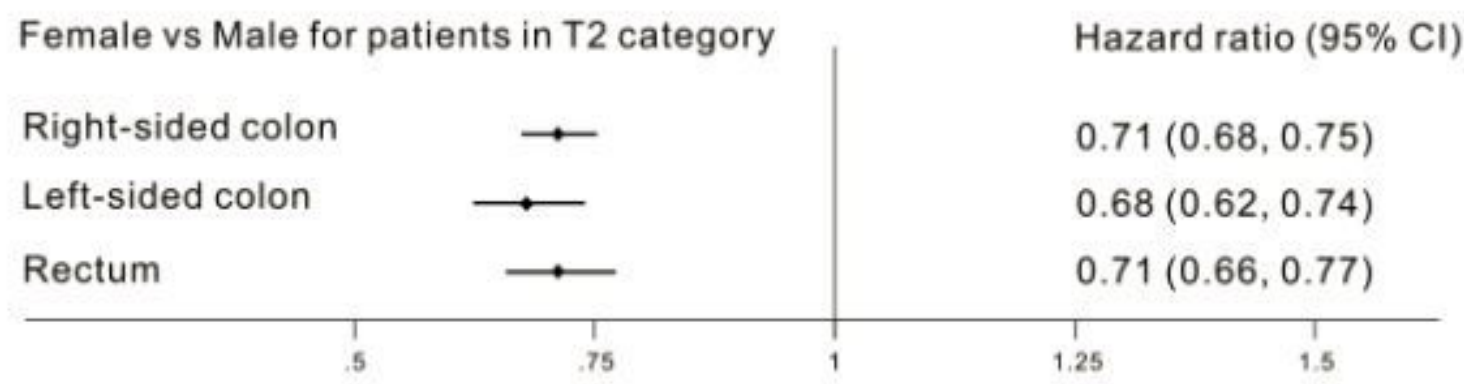

C

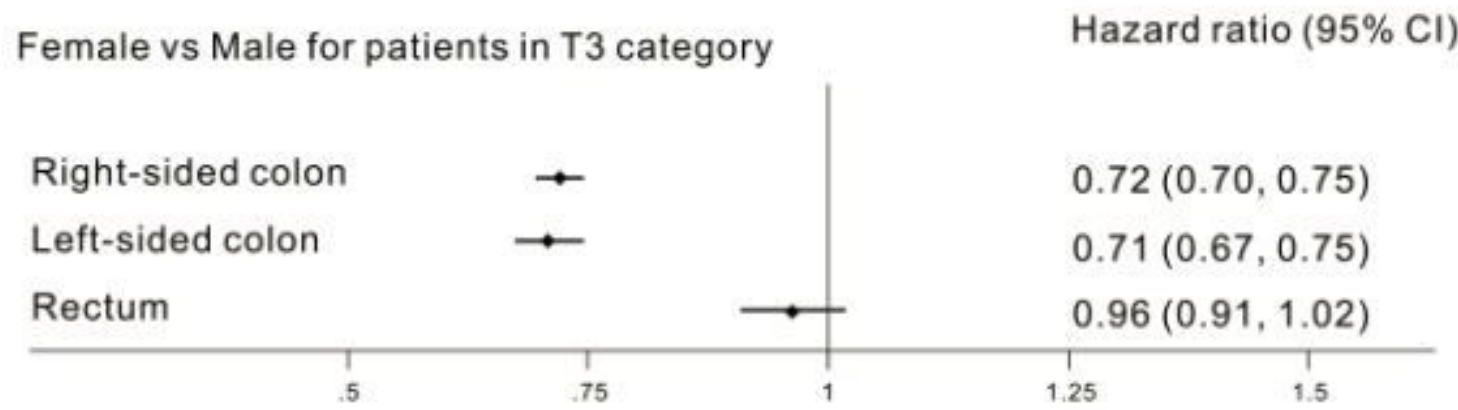

\section{Figure 3}

Forest plot of hazard ratios (HR) and 95\% Cl in OS different subgroups (Cox proportional hazards model analysis) between female and male. (A) Female and male for patients in T1 category; (B) Female and male for patients in T2 category; (C) Female and male for patients in T3 category. 

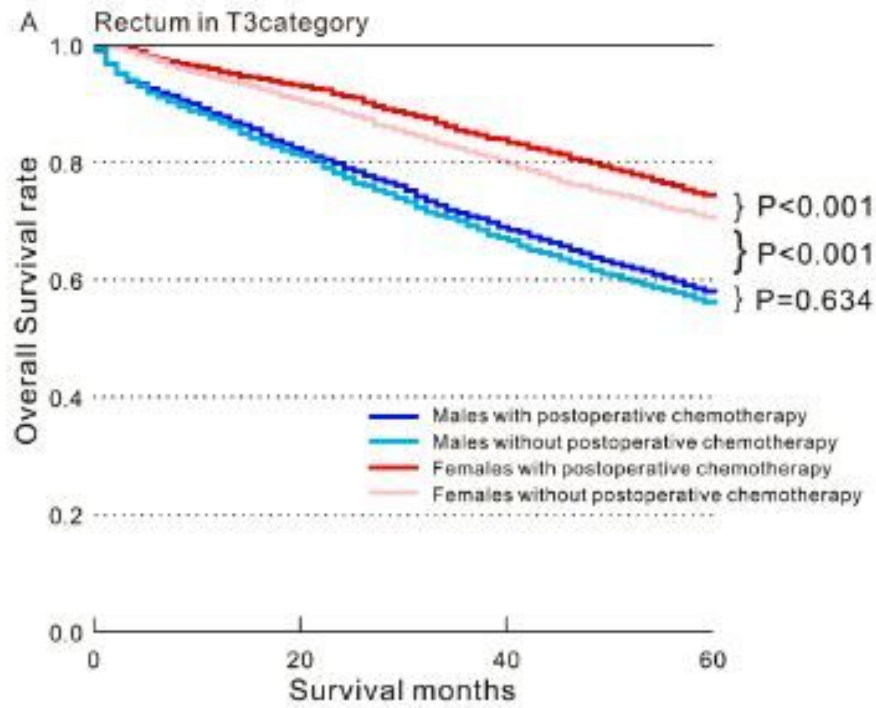
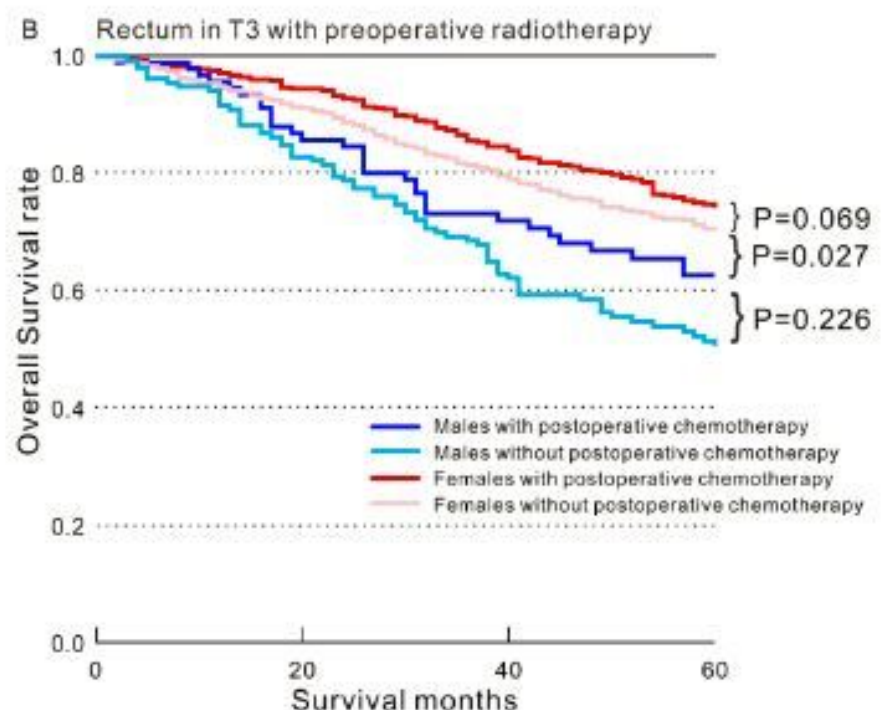

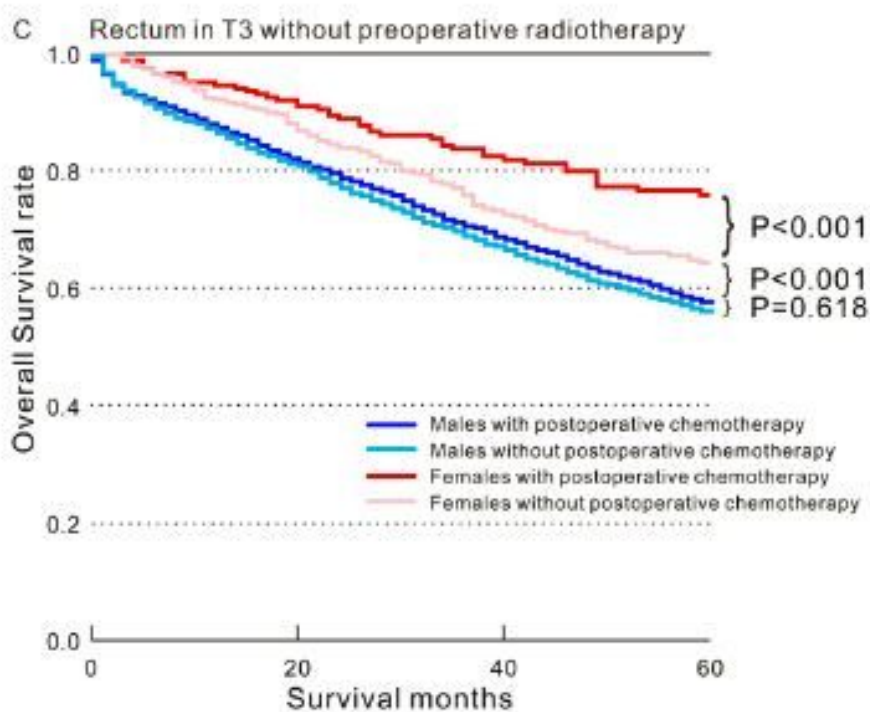

\section{Figure 4}

Kaplan-Meier comparison of overall survival among rectum in T3 category. (A) Rectum in T3 category; (B) Rectum in T3 category with preoperative radiotherapy; (C) Rectum in T3 category without preoperative radiotherapy.

\section{Supplementary Files}

This is a list of supplementary files associated with this preprint. Click to download.

- SupplementalTable4.docx

- SupplementalTable3.docx

- SupplementalTable2.docx

- SupplementalTable1.docx 